\title{
The Equation of Motion of an Electron
}

\author{
R. F. O'Connell* \\ Department of Physics and Astronomy, Louisiana State University \\ Baton Rouge, Louisiana, 70803-4001, USA
}

(October 23, 2018)

\begin{abstract}
The claim by Rohrlich that the Abraham-Lorentz-Dirac equation is not the correct equation for a classical point charge is shown to be incorrect and it is pointed out that the equation which he proposes is the equation derived by Ford and O'Connell for a charge with structure. The quantum-mechanical case is also discussed.
\end{abstract}

*E-mail Address: rfoc@rouge.phys.lsu.edu 


\section{INTRODUCTION}

The equation of motion of a radiating electron is a subject of recurring interest, and in particular, is discussed in the well-known book by Jackson [1]. For a classical point electron, Jackson used the Larmor formula for the total power radiated by an accelerated charge, in conjunction with conservation of energy arguments, to show that the correct equation is the Abraham-Lorentz-Dirac (ALD) equation. But, as pointed out by Jackson, this equation has many well-known unsatisfactory features. This led Rohrlich [2] to question its validity. However, the fact that an equation has unsatisfactory features does not in itself mean that it is inconsistent with the assumptions underlying the derivation. In fact, Rohrlich does not point out anything wrong in the derivation presented in Jackson [1]. The fact is there is nothing wrong!

Moreover, the equation which he proposes has various inconsistencies when applied to a point electron, as was correctly pointed out by Ribaric and Sustersic [3] (a reply by Rohrlich [4] to this paper was unconvincing) and by Baylis and Huschilt [5]. Actually, these criticisms did not go far enough because they failed to point out that the equation proposed by Rohrlich to describe a point charge actually describes a charge with structure! In fact the equation analyzed by both [3] and [5] is not actually the relativistic Landau-Lifshitz equation but Rohrlich's approximate modification of this equation. As we point out below, the Landau-Lifshitz equation itself was obtained by a series of approximations so there is no way of discerning what they correspond to physically except to say that it is definitely not a point particle. This, coupled with the fact that both of these equations are non-linear in the electromagnetic field tensor appears to me to be the source of some of the inconsistencies pointed out by [3] and [5].

Not only does Rohrlich's paper display inconsistencies but it mischaracterizes previous work on this subject in that he claims that his alternative equation to the ALD equation was obtained "- by Ford and O'Connell - as an approximation -." This is incorrect; Ford and O'Connell [6] obtained this exactly! Moreover, they showed exactly that it pertains to 
a particle with structure and not to a point electron, as claimed by Rohrlich. Moreover, in the particular case of the point electron limit, they have, in essence, obtained an exact derivation of the ALD equation for a classical point charge starting with the universallyaccepted Hamilton of non-relativistic quantum electrodynamics(QED). For these reasons and also because others do not seem to have appreciated this point, we feel that it will be instructive to summarize and extend the work of Ford and O'Connell, to point out the nature of the problems with the Rohrlich paper and to outline how the EXACT classical results, for both a point electron and an electron with structure, may be obtained not only from the classical limit of the quantum results but also from an ab initio classical derivation.

Moreover, since many important aspects of the problem arising from quantum and fluctuation effects have been completely ignored in recent discussions, we proceed in the following sections, in showing the development from the classical case of a free electron to the quantum case of an electron with structure, in a potential and subject to both classical (temperature) fluctuations and quantum fluctuations. Only retardation has been neglected. In particular, we attempt to point out clearly results which have been derived in a precise and exact manner from carefully stated initial assumptions $[1,6]$, in contrast to what I refer to as "guesstimates" based on approximations which change the physical nature of the problem in an unknown manner [2].

\section{NONRELATIVISTIC CLASSICAL FREE ELECTRON}

We first note, as points of reference, the classical Newtonian equation of motion for a particle of mass $M$ under the action of an external force $f(t)$

$$
M \ddot{x}=f(t),
$$

the corresponding $\mathrm{AL}$ equation for an electron of charge $e$ 


$$
M \ddot{x}-M \tau_{e} \dddot{x}=f(t),
$$

and the exact equation derived by Ford and O'Connell [6] for a classical electron with a specific structure, dipole interacting with the electromagnetic field,

$$
M \ddot{x}=f(t)+\tau_{e} \dot{f}(t)=f_{\text {eff }}(t) .
$$

Here, a dot denotes differentiation with respect to $t$ and

$$
\tau_{e}=\frac{2}{3} \frac{e^{2}}{M c^{3}}=6 \times 10^{-24} s
$$

First of all, it should be emphasized that there is no conflict between (1) and (2). In particular, there are at least four different but exact derivations for the case of a point electron all of which lead to the same AL equation. They are

(i) the well-known conventional derivation given in Jackson [1].

(ii) the point-electron classical limit of the exact Ford-O'Connell results, given in (6) below (iii) the point-electron limit of a purely classical derivation based on solutions of Maxwell's equations which was presented by Ford in a comprehensive encyclopedia article [7].

(iv) the point-electron limit of a derivation in which the electromagnetic field is treated as a classical stochastic field [see discussion in the paragraph following (7) below].

Three points are immediately clear:

(a) Because of the smallness of $\tau_{e}$, given in (4), the radiative reaction terms given in (2) and (3) are both small compared to the other terms.

(b) There is no conflict between (2) and (3) because they describe different scenarios viz. a point charge and a charge with structure, respectively.

(c) Since from (1) we have that 


$$
M \dddot{x}=\dot{f}(t), \text { to order } \tau_{e}
$$

it follows that equations (2) and (3) only differ from each other by terms of order $\tau_{e}^{2}$. In other words, the equations of motion for a point electron and an electron with structure differ only by terms of order $\tau_{e}^{2}$ and higher order [the exact difference being displayed in (16) below].

Now, because (2) existed since the 1903-1904 period and because it was well-recognized that it had serious shortcomings [1,8,9], many attempts were made to modify it. [1,10,11]. In particular, one proposed modification [10] was, in effect, to add small $\tau_{e}^{2}$ terms to (2) to obtain (3).

However, as we emphasized in [6], none of these prior investigators derived (3) from first principles. Moreover, they surely knew that such additions to the AL equation of motion clearly modified the basic assumptions underlying the AL derivation as they were careful to avoid claiming that the resulting equations represented the exact equation for a classical point electron. Rohrlich, on the other hand, made this unwarranted claim. In fact, his "derivation" of (3) from (2) is, in essence, no different than other ad hoc approaches; he uses the word "exact" liberally but glosses over the fact that, in the discussion immediately following and referring to his equation (3), he states that" - Since the latter is a small term, the acceleration in it can be replaced by the approximate expression from -." In fact, he is using a "guesstimate" and he is simply working to order $\tau_{e}$ ! In essence, he is adding a term of order $\tau_{e}^{2}$ without realizing that he is changing the physics of the problem from a point electron model to a model with structure.

The key question to be answered is whether or not (3) can be derived exactly and what new physics does it incorporate. The answer is that this has already been done [6]. In [6] we presented an exact quantum mechanical result for the equation of motion of the radiating electron from which we obtained (3). Our starting point $[12,13]$ was the universally accepted non-relativistic Hamiltonian of QED describing an electron dipole interacting with 
a radiation field and in an arbitrary potential but generalized to include an arbitrary form factor $f_{k}$ (Fourier transform of the electron charge distribution) and also allowing for the presence of a time-dependent external field. Next, we used the Heisenberg equations of motion to obtain equations of motion for the dynamical variables of the electron and of the radiation field in terms of each other. This enabled us to write down the equation of motion of an electron with charge $e$ and bare mass $m$, dipole interacting with the electromagnetic field and moving in a potential $V(r)$, in the form of a generalized quantum Langevin equation $[12,13]$

$$
m \ddot{x}(t)+\int_{-\infty}^{t} d t^{\prime} \mu\left(t-t^{\prime}\right) \dot{x}\left(t^{\prime}\right)+V^{\prime}(x)=F(t)+f(t)
$$

where $m$ is the bare mass, $x(t)$ is the coordinate operator, $F(t)$ is the operator-valued random (fluctuating) force, $f(t)$ is the external force, $\mu(t)$ is the memory function and where the dot and prime denote, respectively, the derivative with respect to $t$ and $x$. This is an exact result and explicit values are known for $\mu(t)$ and $F(t)$ in terms of the parameters of the heat bath (in this case the radiation field). If fact, the (three-dimensional) random force is simply the free-field operator [13] i.e.

$$
\vec{F}(t)=-e \bar{E}(t)
$$

where $\bar{E}(t)$ is the electric field associated with the radiation field. In fact, such a term is crucial in order to satisfy the fluctuation-dissipation theorem [9].

Equation (6) is extremely general in that the choice of the electron charge distribution has not been specified. In particular, specific choices for $\mu(t)$ lead to a variety of equations of motion, in particular (2) and (3). Furthermore, the corresponding classical equation has exactly the same form as (6) but now the dynamical variables are all classical quantities. In fact, it is of interest to indicate how the classical results can be obtained ab initio. Completely analogous to the derivation outlined above, we start with the classical Hamiltonian of 
electrodynamics. Next, we use Poisson bracket equations of motion and obtain an equation of motion which is exactly the same form as (6) but now all the quantities are c-numbers. It should be emphasized that there are also fluctuations in the classical case (due to temperature), whereas in the quantum case, there are both temperature and quantum fluctuations. Thus, it is clear that the quantum and classical cases can be discussed in tandem.

Among other things, one advantage of the approach which we have adopted is that it enables us to make use of the powerful techniques of stochastic physics. In particular, denoting the Fourier transform of $\mu(t)$ by $\tilde{\mu}(\omega)$, it was shown $[13,6]$ that it is analytic in the upper half of the $\omega$ plane and that it has a positive real part. This is equivalent to the demand $[12,13]$ that all the poles of $\alpha(\omega)$, the generalized susceptibility, must be in the lower half of the complex plane (as otherwise the principle of causality is violated). It immediately follows [6] that the AL point electron solution does not fulfil these criteria and that we must consider an electron with structure.

To proceed further, it is necessary to choose a particular form factor (but, as outlined below, it can be shown that our results are correct to order $\tau_{e}$ for all reasonable choices) and we chose an electron form-factor [6], $\Omega^{2} /\left(\Omega^{2}+\omega^{2}\right)$, ̀̀ la Feynman [15], with a shape convenient for calculation but arbitrary in the sense that it depends on the choice of $\Omega$, a large cut-off frequency. Also, as is generally the case in quantum field theory, mass renormalization is also required and we found that the renormalized mass $M$ is given in terms of the bare mass $m$ by the relation

$$
M=m+2 e^{2} \Omega / 3 c^{3}=m+\tau_{e} \Omega M
$$

Choosing $\Omega \longrightarrow \infty$ would correspond to the case of a point electron. But, as is clear from (8), $\Omega>\tau_{e}^{-1}=1.60 \times 10^{23} s^{-1}$, would require a negative rest mass and, based on the analyticity arguments given above, this is precluded by physical considerations. We conclude that

$$
\Omega \leq \tau_{e}^{-1}
$$


Thus, the choice $\Omega=\tau_{e}^{-1}$ corresponds to $m=0$ and describes an electron with the smallest size consistent with causality. The size is of the order of the classical electron radius [16], underlining the fact that the dipole interaction is more than adequate to describe the basic physics. [Nevertheless, it would be of interest to calculate possible retardation modifications [14] to (6), a topic we hope to consider in the future.] This choice gives

$$
f_{k}^{2}=\frac{1}{1+\omega^{2} \tau_{e}^{2}}
$$

with the result that the exact equation of motion reduces to

$$
M \ddot{x}(t)+V_{e f f}^{\prime}(x)=F_{e f f}(t)+f_{e f f}(t),
$$

where

$$
f_{\text {eff }}(t) \equiv f(t)+\tau_{e} \dot{f}(t)
$$

and, similarly, for the other "effective" quantities.

In summary, based on the choice of form factor given by $(10),(11)$ is an exact quantum mechanical result for an electron with a minimum size (which turns out to be of the order of the classical electron radius [16]) consistent with causality. However, we will defer discussion of the quantum aspects until Sec. 3. We will now examine some interesting limiting cases. (i) Classical Limit (with $V=0$ )

The result has the same form as (11) but with the operator quantities replaced by c-number quantum mechanical averages and with $V=0$. Hence

$$
M \ddot{x}=f_{\text {eff }}(t)+F_{\text {eff }}(t),
$$

where, as before, $f(t)$, is the external force and the fluctuation force $F(t)=-e E(t)$, where $E(t)$ is the classical electric field associated with the radiation field. Only in the case where one takes an ensemble average so that 


$$
\langle E(t)\rangle=0
$$

does this result reduce to (3). On the other hand, in the absence of an external force, we have

$$
M \ddot{x}=F_{\text {eff }}(t),(\text { if } f(t)=0) .
$$

This stochastic equation is intriguing, since it displays fluctuations (the right hand side is a fluctuating force due to temperature effects which, classically, give zero when the temperature $T=0$ ) with no dissipative term on the left hand side. Nevertheless, the fluctuationdissipation theorem still holds, as discussed in detail in Ref. [9].

Now that we have established that (3) is an exact result for the classical equation of motion of an electron with a specified structure and in the case where $V(x)=0$ and where an ensemble average has been taken, we turn now to some further ramifications:

(ii) Equation (1) may be re-written as an exact equation of motion involving derivatives of $x$ (instead of the single derivative of $f(t)$ ) but it requires an infinite number of terms in powers of $\tau_{e}$ and derivatives of $x[17]$ :

$$
M x^{(2)}+M \sum_{n=3}^{\infty}(-1)^{n} \tau_{e}^{n-2} x^{(n)}=f(t),
$$

where $x^{(n)}$ indicates the $n$ derivative of $x$ with respect to $t$.

(iii) If one again chooses the Feynman form factor but with $\Omega<\tau_{e}^{-1}$ (as distinct from the value of $\Omega=\tau_{e}^{-1}$ chosen above) then higher order terms in $\tau_{e}$ appear on the right-side of (3), as discussed in [6].

(iv) The rate of radiation, $P(t)$ say, associated with the equation of motion (3) is no longer the Larmor rate but, as we proved by two different methods $[18,19]$, we now have

$$
P(t)=\frac{\tau_{e}}{M} f^{2}(t)=\frac{2 e^{2}}{3 c^{3}}[f(t) / M]^{2}
$$


In other words it differs from the Larmor result by terms of order $\tau_{e}^{2}$.

We should emphasize once more that (11) is an exact result corresponding to the particular choice of form factor given by (10) but now we will present a simple argument to show that (11) is actually correct, to a very good approximation, for all physically reasonable choices of electron structure. We proceed by considering another reasonable choice of form factor and also, without loss of generality, we take $V(x)=0$. Thus, for example, the choice

$$
f_{k}^{2}=\frac{\Omega^{4}}{\left(\omega^{2}+\Omega^{2}\right)^{2}}
$$

and $\Omega=2 \tau_{e}^{-1}$, corresponding to a sharper cut-off, leads to

$$
M \ddot{x}(t)=\left(1+\frac{\tau_{e}}{2} \frac{d}{d t}\right)^{2}\{F(t)+f(t)\} .
$$

Also, the typical heat bath correlation time, which is a measure of the time-scale over which $F(\tau)$ changes on the average, is $[13] \hbar /(2 \pi k T)=\left(1.1 \times 10^{-12} / T\right) s^{-1}$, which is very large

compared to $\tau_{e}=6 \times 10^{-24} \mathrm{~s}$. Thus, it is an excellent approximation to consider only lowest order in $\tau_{e}$, in which case we recover (13). It is clear that, by a similar argument, all physically reasonable choices of electron structure lead to (13).

Thus, to lowest order in $\tau_{e}$ (an excellent approximation), (13), and more generally (11), are model-independent results for all choices of electron structure fulfilling reasonable physical criteria.

\section{NONRELATIVISTIC QUANTUM MECHANICAL EQUATION}

The exact result for an arbitrary distribution of charge is given by (6) with all the dynamical variables to be regarded as operator quantities. For the particular choice of form factor given by (10), the resultant equation is (11) i.e. it has the same form as the classical equation. Also, when $V=0$ and $f(t)=0$, the resultant equation has the same form as 
(15) but there is a crucial difference in the quantum case viz. the right-side of (15) now contains quantum fluctuations as well as temperature fluctuations. Thus, even at $T=0$, there is a contribution from the ubiquituous zero-point oscillations of the electromagnetic field (viz. the same fluctuations that give use to the Lamb shift and the Casimir effect)! Furthermore, one may verify that such fluctuation terms are essential to ensuring that the correct quantum-mechanical commutation relations for the dynamical variables hold.

Finally, returning once more to our general quantum-mechanical result (11), it is instructive to consider the case of an electron in the oscillator potential

$$
V(x)=\frac{1}{2} K x^{2}
$$

It then follows [see (12) and the following line] that

$$
\begin{aligned}
V_{e f f}(x) & =\frac{1}{2} K\left\{x^{2}+\tau_{e} \frac{d\left(x^{2}\right)}{d t}\right\} \\
& =\frac{1}{2} K x^{2}+K \tau_{e} x \dot{x} .
\end{aligned}
$$

Thus, we obtain exactly the equation of motion.

$$
M \ddot{x}+\zeta \dot{x}+K x=-e E(t)
$$

where $\zeta=K \tau_{e}$. The presence of an Ohmic-type dissipation term is now manifest. In fact, this term is exactly of the form universally used in practical applications of the AL equation to radiation damping problems (where it is derived as a weak coupling approximation to the dissipation term [1]). It is also of interest to note that $\zeta / \sqrt{K M}$ can be identified as a dimensionless measure of the weak coupling (of the order of $10^{-7}$ for optical frequencies) of relevance for studies in areas such as quantum optics. 


\section{RELATIVISTIC EQUATION OF MOTION}

Analogous to Dirac's extension of the AL equation to the relativistic domain, we also carried out an analogous extension of (3) with the result [20]

$$
\begin{aligned}
M a^{\mu}= & \frac{e}{c} F^{\mu}{ }_{\kappa} u^{\kappa} \\
& +\tau_{e} \frac{e}{c}\left(\frac{d}{d \tau} F_{\lambda}^{\mu} u^{\lambda}-\frac{1}{c^{2}} u^{\mu} u^{\kappa} \frac{d}{d \tau} F_{\kappa \lambda} u^{\lambda}\right),
\end{aligned}
$$

where

$$
u^{\mu}=\frac{d x^{\mu}}{d \tau}, a^{\mu}=\frac{d u^{\mu}}{d \tau}
$$

with

$$
\begin{aligned}
d \tau & =\frac{1}{c} \sqrt{g_{\kappa \lambda} d x^{\kappa} d x^{\lambda}}=\sqrt{1-v^{2} / c^{2} d t} \\
& =\frac{1}{\gamma} d t .
\end{aligned}
$$

Also, the field tensor has the following form when expressed in terms of the laboratory electric and magnetic fields,

$$
F^{\mu v}=\left(\begin{array}{rrrr}
0 & -E_{x} & -E_{y} & -E_{z} \\
E_{x} & 0 & -B_{z} & B_{y} \\
E_{y} & B_{z} & 0 & -B_{x} \\
E_{z} & -B_{y} & B_{x} & 0
\end{array}\right) .
$$

It is also of interest to note that our equation of motion (3) can be written in the three-vector form

$$
M \frac{d \gamma \vec{v}}{d t}=\vec{F}+\tau_{e}\left[\gamma \frac{d \vec{F}}{d t}-\frac{\gamma^{3}}{c^{2}}\left(\frac{d \vec{v}}{d t} \times(\vec{v} \times \vec{F})\right)\right]
$$

where 


$$
\vec{F}=e(\vec{E}+\vec{v} \times \vec{B})
$$

is the Lorentz force. In the quantum mechanical case, there will also be relativistic fluctuation terms.

Our relativistic form (23) followed from the non-relativistic result (3) by simply replacing $\ddot{x}$ by $a^{\mu}$ and $f(t)$ by

$$
f^{\mu}=\frac{e}{c} F_{\kappa}^{\mu} u^{\kappa}
$$

where $F^{\mu}$ is the external electromagnetic field tensor. Also $\dot{f}(t)$ is replaced by

$$
g^{\mu}=\dot{f}^{\mu}-\frac{1}{c^{2}} u^{\mu} u^{\kappa} \dot{f}_{\kappa}
$$

to ensure that

$$
g^{\mu} u_{\mu}=0
$$

which is valid for any force four-vector.

Landau and Lifshitz also have written down a classical relativistic equation [11] but it can be classified as a "guesstimate" in that it is derived from the Dirac relativistic result by a series of approximations. In other words, their starting point is based on the result for a point particle. They then use a series of iterations (approximations) to express the result directly in terms of the field tensor but, of course, these changes correspond to a change in the underlying physics without any knowledge as to what these changes are. Thus their resultant equation has no discernable special significance despite Rohrlich's claim [2], and, of course, it should be emphasized that Landau and Lifshitz never made such a claim. It is interesting to note that the Landau-Lifshitz equation is non-linear in the field tensor but 
whether or not non-linear terms will appear in the ultimate theory is a matter of conjecture at this stage. Also, by construction, it is purely classical (with $T=0$ ) and thus does not have fluctuation terms.

It clearly would be desirable to develop a relativistic theory ab initio but incorporating electron structure into such a theory constitutes a formidable task. A start in that direction

has been made by Johnson and $\mathrm{Hu}[21]$ whose starting-point was the action describing the interaction of a relativistic free particle interacting with a quantum scalar field (as distinct from the quantum electrodynamic field). Then, using stochastic methods, these authors derived a relativistic Langevin equation leading to causal solutions in the non-relativistic limit and they obtained results consistent with our results. Of course, as Johnson and $\mathrm{Hu}$ point out, some of the results obtained are features of the assumption of a scalar field but they certainly motivate their ultimate goal to extend the work to the relativistic QED case.

\section{ACKNOWLEDGMENTS}

The author is pleased to acknowledge a long-time collaboration with G. W. Ford which led to the essential results presented here. 


\section{REFERENCES}

[1] J. D. Jackson, Classical Electrodynamics, 3rd ed. (Wiley, New York 1998) Chap. 16.

[2] F. Rohrlich, Phys. Lett. A 283 (2001) 276; ibid. A 303 (2002) 307.

[3] M. Ribaric and L. Sustersic, Phys. Lett. A 295 (2002) 318.

[4] F. Rohrlich, Phys. Lett. A 295 (2002) 320.

[5] W. E. Baylis and J. Huschilt, Phys. Lett. A 301 (2002) 7.

[6] G. W. Ford and R. F. O'Connell, Phys. Lett. A 157 (1991) 217.

[7] G. W. Ford, "Electromagnetic Radiation" in Encyclopedia of Applied Physics, Vol. 5 (VCH Publishers, Inc. 1993), Sec. 5.

[8] R. F. O'Connell, "Dissipative and Fluctuation Phenomena in Quantum Mechanics with Applications", in Festschrift for J. P. Dahl, Int. J. Quantum Chem. 58 (1996) 569.

[9] G. W. Ford and R. F. O'Connell, Phys. Rev. A 57 (1998) 3112.

[10] C. J. Eliezer, Proc. R. Soc. A 194 (1948) 543.

[11] L. Landau, E. Lifshitz, The Classical Theory of Fields, Pergamon Press, New York, 1975 .

[12] G. W. Ford, J. T. Lewis, and R. F. O'Connell, Phys. Rev. Lett. 55 (1985) 2273.

[13] G. W. Ford, J. T. Lewis, and R. F. O'Connell, Phys. Rev. A 37 (1988) 4419.

[14] E. J. Moniz and D. H. Sharp, Phys. Rev. D 15, (1977) 2850.

[15] R. P. Feynman, Phys. Rev. 76 (1949) 769.

[16] R. F. O'Connell, in Festschrift for A. O. Barut, Foundations of Physics 23 (1993) 461.

[17] G. W. Ford and R. F. O'Connell in Festschrift for H. Walther, Appl. Phys. B 60 (1995) 301. 
[18] G. W. Ford and R. F. O'Connell, Phys. Lett. A 158 (1991) 31.

[19] G. W. Ford and R. F. O'Connell Phys. Rev. A 44 (1991) 6386.

[20] G. W. Ford and R. F. O'Connell, Phys. Lett. A 174 (1993) 182.

[21] P. R. Johnson and B. L. Hu, Phys. Rev. D 65 (2002) 065015. 